\title{
The in situ Studies on the Anomalous Domain Switching Caused by Trace Amount of Oxygen Vacancies
}

\author{
Shaobo Cheng ${ }^{1}$, Qingping Meng ${ }^{1}$, Myung-Geun $\mathrm{Han}^{1}$, Shiqing Deng ${ }^{2}$, Xing $\mathrm{Li}^{3}$, Gianluigi A. Botton ${ }^{4}$, \\ and Yimei $\mathrm{Zhu}^{1 *}$ \\ ${ }^{1 .}$ Department of Condensed Matter Physics and Materials Science, Brookhaven National Laboratory, Upton, NY, \\ USA. \\ 2. School of Materials Science and Engineering, Tsinghua University, Beijing, P. R. China. \\ 3. Department of Engineering Physics, Zhengzhou University, Zhengzhou, Henan, P. R. China. \\ 4. Canadian Centre for Electron Microscopy and Department of Materials Science and Engineering, McMaster \\ University, Hamilton, ON, Canada. \\ * Corresponding author: zhu@bnl.gov
}

Multiferroic materials, especially single phase multiferroic materials, have aroused worldwide interest because of their fascinating physics and the great potential device applications [1-3]. $\mathrm{YMnO}_{3}$ is a typical single phase multiferroic material, which has room temperature ferroelectricity and low temperature antiferromagnetism. Since it has hexagonal symmetry, six-fold vortex cores are expected. However, with the introduction of the line defects, non-six-fold vortex cores can form and be detected [3]. Here, we have studied the intriguing domain switching behaviors of $\mathrm{YMnO}_{3}$ single crystalline sample with small amount of point defects, i.e. oxygen vacancies [4].

Transmission electron microscopy (TEM) has demonstrated itself to be a powerful tool, capable for versatile structural characterizations in nanoscale [5]. Here, by taking advantage of the in situ TEM technique, we have successfully applied external biasing up to $180 \mathrm{kV} / \mathrm{cm}$ onto the $\mathrm{YMnO}_{3}$ samples prepared by the focused ion beam (FIB) for making the ferroelectric domain switching. Surprisingly, the ferroelectric domain switching only happened at the topmost surface and a stripe like domain showed up at the top surface as shown in Figure 1(d). With higher external biasing, the width of the surface domain-switching area expand more but we can never make the whole sample switched (Shown in Figure 2(a)-(e)). Since our single crystalline $\mathrm{YMnO}_{3}$ sample was made by floating zone method in $\mathrm{Ar}$ atmosphere, it is inevitable to have oxygen vacancies formed in the sample. We thus speculate that the oxygen vacancies play an important role in this unusual domain switching phenomenon.

Further Landau based numerical calculations results show that the tiny amount of oxygen vacancies can strongly influence the distribution of the electric field inside of the sample. The external biasing can only be applied at the topmost surface. The penetration depth of the electric biasing has an almostlinear-like relationship with the external bias. Besides, considering the width of the switched domain in the experiments, we can even evaluate the density of the carriers in samples. In our case, the density of carriers inside of our single crystalline $\mathrm{YMnO}_{3}$ is calculated to be between $10^{23} / \mathrm{m}^{3}$ and $10^{24} / \mathrm{m}^{3}$.

In conclusion, by taking advantage of in situ TEM experiments and numerical calculations, we have studied the unusual domain switching behaviors with quantitative analyses. The trace amount of oxygen vacancies can dramatically affect the domain switching behaviors and confine the switching phenomenon only at the top surface of the samples. The oxygen vacancies could result in the redistribution of the electric potential inside of the samples, which could be further validated by the numerical calculations. Moreover, by combining the experiments and theoretical calculations, we could have an estimation of the carrier densities within the samples as well. We hope our exciting results will attract more zealous researchers to further explore the fascinating physics beneath the multiferroic 
materials and pave the way towards the cutting-edge structure design for modern functional devices applications [6].

\section{References:}

[1] S Cheng et al., Science Advances 4 (2018), eaar4298.

[2] X Li et al., Nanomaterials 8 (2018), p. 188.

[3] S Cheng et al., Physical Review Letters 118 (2017), p. 145501.

[4] S Cheng et al., Advanced Electronic Materials (2019), p. 1800827.

[5] X Li et al., Advanced Materials Interfaces 6 (2018), p. 1701246.

[6] The electron microscopy work was carried out at Brookhaven National Laboratory and supported by the U.S. DOE Basic Energy Sciences, Materials Sciences and Engineering Division under Contract No.

DESC0012704.

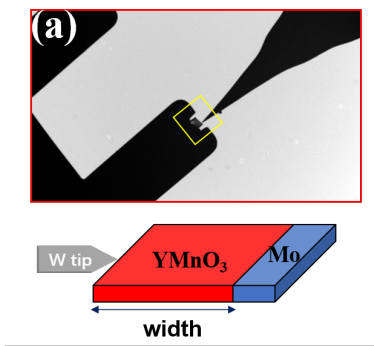

(b)
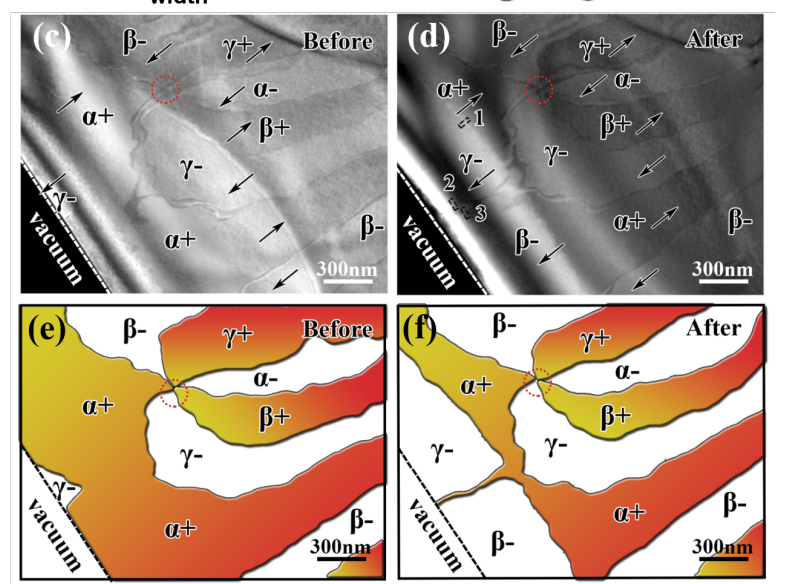

Figure 1. Experimental setup for in situ transmission electron microscopy (TEM) studies. (a) Low magnification TEM image showing the in situ experimental configuration. A schematic diagram for the experimental setup is shown at the bottom. A tungsten tip is making contact with the $\mathrm{YMnO}_{3}$ sample. (b) Hexagonal cell of $\mathrm{YMnO}_{3}$. The $\mathrm{Y}, \mathrm{Mn}$ and $\mathrm{O}$ atoms are in deep green, purple and red, respectively. (c) Dark field TEM image showing the domain configuration before applying the bias. (d) Dark field TEM image showing the domain after biasing. (e)(f) Schematic drawing of the domain configurations for (c) and (d), respectively.
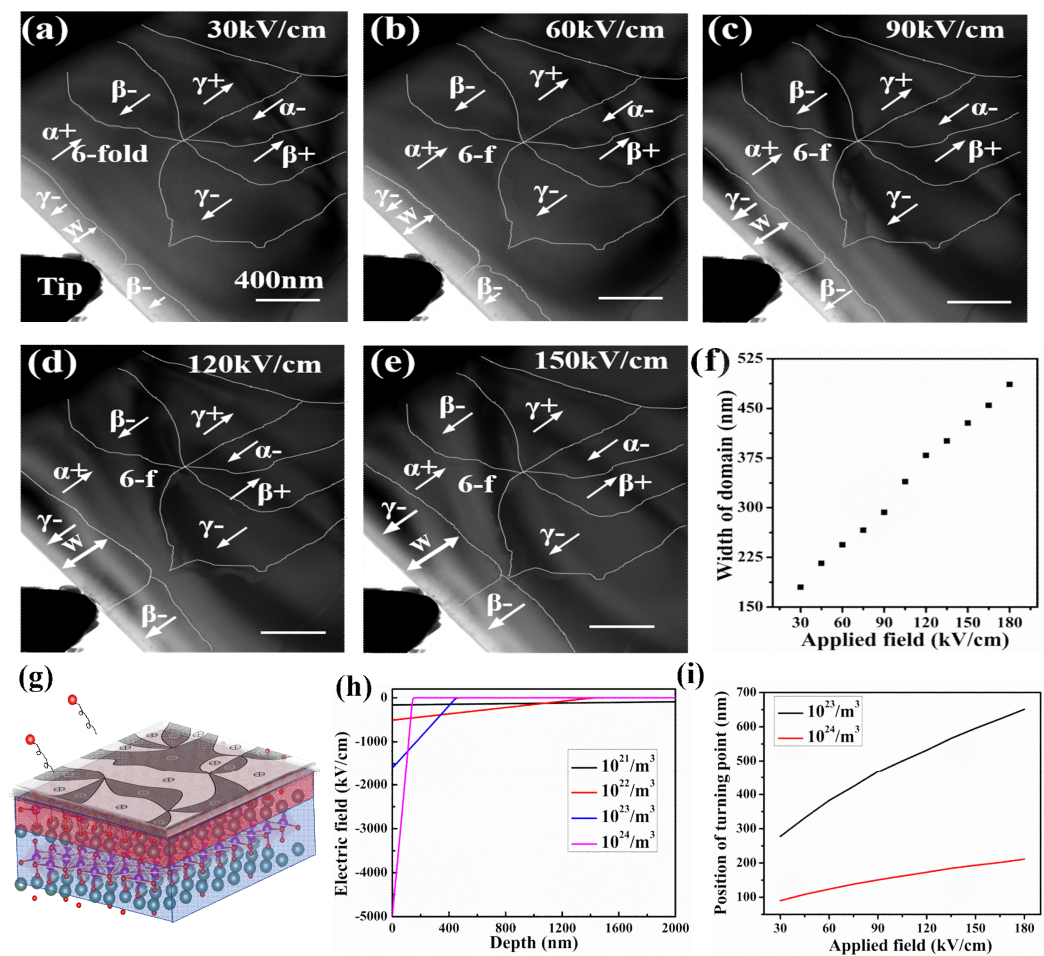

Figure 2. The in situ biasing TEM experiments and numerical calculations results of the $\mathrm{YMnO}_{3}$ sample. (a)-(e) TEM images showing the domain configurations under different external electric fields. A stripe like domain shows up and expands when increase the electric fields. (f) The relationship between the applied external field and the width of the stripe like domain. (g) Illustration of the domain switching for $\mathrm{YMnO}_{3}$ with trace amount of oxygen vacancies. The top surface shows a typical domain configuration of $\mathrm{YMnO}_{3}$ at (001) surface. The red and blue boxes represent the surface area and interior area of the sample. Two oxygen atoms escape, indicating the sample has oxygen deficiency. (h) Numerical calculations showing the relationship between electric field and sample thickness. The electric field is almost zero inside of the sample. The position where electric field changes to zero is called "Position of turning point". (i) The calculated results showing the relationship between the position of turning point and sample thickness. Different carrier densities will lead to different penetration depths of electric field. 